\title{
Stimulus complexity, free looking time, and inspective exploration
}

\author{
JUDITH A. GASCHK, B. L. KINTZ, 1 AND RICHARD W. THOMPSON \\ WESTERN WASHINGTON STATE COLLEGE
}

\begin{abstract}
Fourteen Ss rated 59 objects on a seven point complexity scale. From these ratings 10 objects were chosen at the high, middle, and low levels of complexity, and $35 \mathrm{~mm}$ slides were made of these 30 objects. Fifteen $S$ s manipulated and inspected each of the objects for as long as they wished and a second group of $15 \mathrm{Ss}$ viewed slides of the objects for as long as they wished. Analysis of the time scores from these two groups revealed that both inspective manipulation time and free looking time increased as a function of stimulus complexity. Inspective manipulation time was greater than free looking time except at the low complexity level.
\end{abstract}

Several recent experiments have used free looking time as a measure of attention and exploratory behavior. These studies indicate that the duration of attention or exploratory behavior is a function of stimulus variables (Berlyne, 1957, 1958; Leckart, 1966, 1967; Leckart \& Bakan, 1965). A consistent finding has been that as stimulus complexity increases, the duration of free looking time increases.

Berlyne (1960) has suggested that if an $S$ is permitted to pick up an object and turn it around, the amount of exploration might be greater than if the $S$ merely looked at the stationary object or a picture of it. Such inspective exploration of an object may reveal hidden aspects of the object, both external and internal, which are not seen when merely viewing the object.

Welker, in a series of studies with chimpanzees (1956a, b, c), has found that the amount of time Ss spent manipulating an object was related to the complexity of the object. These data seem to support the suggestion of Berlyne.

The present experiment was undertaken to examine the effect of stimulus complexity on free looking time and inspective manipulation time. It was expected that both free looking time and inspective manipulation time would increase as stimulus complexity increased, and that Ss would spend more time in inspective manipulation of objects than in looking at pictures of the objects.

\section{METHOD}

The experiment was performed in two parts. In the first part 14 Ss, volunteers from an introductory psychology class, individually rated each of 59 objects on a seven-point complexity scale. Each S was handed each object and allowed to look at it as long as he wished. He was asked to make a judgment of its complexity, on a seven-point scale, and to ignore all other characteristics of the object. No attempt was made to define complexity for the Ss. The objects were items small enough to be handled easily and consisted of such things as a plastic sponge, a child's puzzle, an electric stepping switch, a partially disassembled clock, a shuttlecock, etc. A mean complexity rating was computed for each object, and 30 objects were chosen, 10 each at the high, middle, and low levels of complexity. The means and standard deviations for the three sets of complexity ratings were: high, 5.53 and 0.63 ; middle, 2.80 and 0.17 ; low, 1.71 and 0.24 . A $35 \mathrm{~mm}$ photographic slide was made of each object and the objects and their corresponding slides were used in the second part of the experiment.

\section{Subjects}

The Ss were 15 male and 15 female volunteers from an introductory psychology class.

\section{Apparatus}

The experiment was performed in a sound-deadened room with a one-way window for viewing the Ss. A large comfortable chair was positioned 37 in. from, and facing, a white screen. A Kodak Carousel 650 projector was located 60 in. from the screen and was behind the S's chair. The projector presented an $18 \times 27$ in. image on the screen. $S$ was provided with a switch which automatically changed the slide and activated a BSR Electronics print-out counter located in an adjacent room.

\section{Procedure}

The $30 \mathrm{Ss}$ were randomly assigned to two groups of 15 each. One group viewed slides of the 30 objects and the other group engaged in inspective manipulation of the objects.

\section{Inspective Manipulation}

Each $S$ was seated in the sound-deadened room and instructed that he would be given a series of objects that he could inspect or manipulate for as long as he wished. When he was finished with one object he could return it to $E$ and he would be given another object. He was told that he would not be asked to remember anything and that he had as much time as he liked. $S$ was then handed an object and allowed to inspect it. A second $E$ in the adjoining room observed $S$ through the one-way window and recorded inspective manipulation time. The objects were presented in a random order and were kept out of S's view until they were presented.

\section{Free Looking Time}

Each $\mathrm{S}$ was seated in the sound-deadened room and told that he could view each of a series of slides for as long as he wished. He could change the slides by pushing a button provided for that purpose. The $S$ was assured that he would not be required to remember anything, $E$ left the room, and the first slide was presented automatically. Each time $S$ changed the slide he also activated the print-out counter which measured the duration of free looking time for each slide. The slides were presented in a different random order for each $\mathrm{S}$.

\section{RESULTS}

The mean free looking time or inspective manipulation time was computed for each $S$ for each of the three levels of stimulus complexity. Figure 1 presents the mean free looking time and inspective manipulation time for slides and objects at the three levels of complexity. These data were analyzed by a Lindquist Type 1 analysis of variance with objects and slides as the between dimension and levels of complexity as the within dimension. There was a significant effect of Slides vs Objects $(F=6.14$, $\mathrm{df}=1 / 28)$, Complexity $(\mathrm{F}=13.83, \mathrm{df}=2 / 56)$, and the interaction of Slides-Objects with Complexity $(F=8.70, \mathrm{df}=2 / 56)$. To clarify the interaction, Treatment by Subjects analyses of variance were computed for both objects and pictures and both revealed a significant effect of complexity.

A further analysis of the slide group showed that the differences between low and medium complexity conditions is not reliable $(t=1.72, \mathrm{df}=28)$, but that the medium and high conditions are different $(t=2.58, \mathrm{df}=28)$, and the low and high conditions are different $(t=4.29, \mathrm{df}=28)$. When the same analyses were done with objects, the same results were found. The low and medium complexity conditions did not differ reliably $(t=1.15, \mathrm{df}=28)$, the medium and high complexity conditions differed $(t=3.25$, $\mathrm{df}=28$ ), and the low and high complexity conditions differed $(\mathrm{t}=4.40, \mathrm{df}=28)$.

The final set of analyses showed that the slide groups did not differ reliably from the object group at the low complexity 


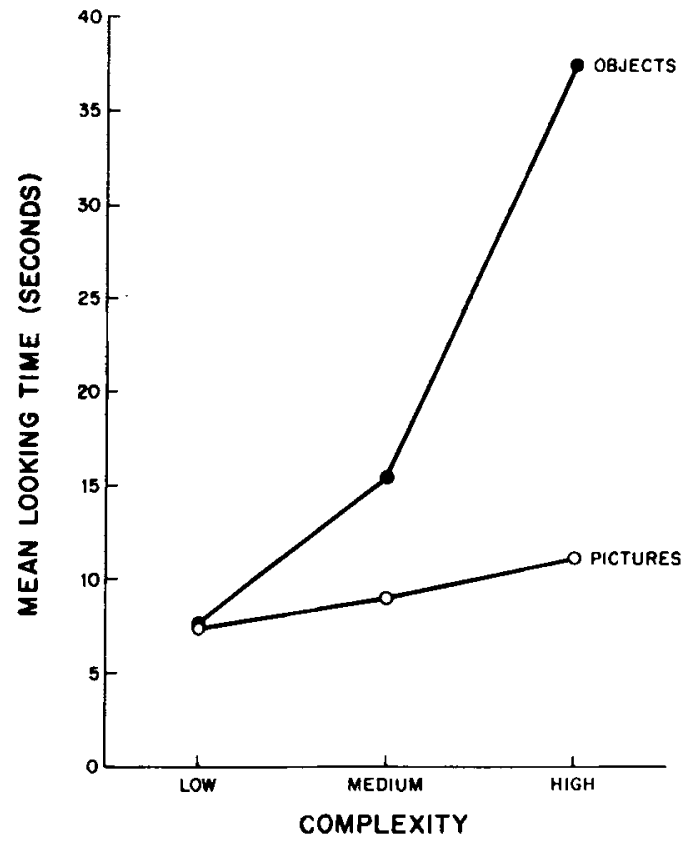

Fig. 1. Mean inspective manipulation time and free looking time as a function of stimulus complexity.

condition $(t=0.14, \mathrm{df}=28)$, but did at the medium complexity condition $(t=8.45, d f=28)$, and at the high complexity condition $(t=34.33, \mathrm{df}=28)$.

\section{DISCUSSION}

The results of the mean free looking times in the present study are consistent with the previous findings of Berlyne (1957, 1958), Leckart (1966), and Leckart and Bakan (1965): looking time increased as the complexity of the slides increased; the manipulation time of the objects also increased with complexity.

We hypothesized that by providing the Ss with the opportunity to manipulate the objects, the overall inspective exploration time would be greater than the slide viewing time, that this difference would increase with complexity. No reliable difference was found between the manipulation time of the low complexity objects and the viewing time of the low complexity slides. The manipulation time for the medium complexity objects was greater than the viewing time for the medium complexity slides, and the same held for the high complexity conditions. These results are consistent with those of Berlyne's position concerning inspective exploration $(1900)$ in that manipulation increased the anount of time spent examining the stimuli. The results are also consistent with the data obtained by Welker (1956a, b, c) with chimpanzees.

One possible explanation for the greater amount of examination time when objects were manipulated could be that the act of taking the object required time, thus producing an artifactual difference. The fact that there was no difference between the object group and the slide group for the low complexity condition, and that the high complexity difference is so huge, seems to be an effective refutation of that explanation.

A more reasonable explanation seems to be that the added sensory dimension of touch increases the effective complexity of the stimuli. If complexity is considered to be the result of some combination of the many stimulus characteristics of the objects, as perceived by the Ss, then the several tactual components would contribute to the total configuration of complexity. Also there are additional stimulus components in the visual modality as the Ss turn the objects to reveal new facets that would be unnoticed or hidden in the two dimensions of a picture.

\section{REFERENCES}

BERLYNE, D. E. Conflict and information-theory variables as determinants of human perceptual curiosity. Journal of Experimental Psychology, 1957, $53,399-404$.

BERLYNE, D. E. The influence of complexity and novelty in the visual figures on orienting responses. Journal of Experimental Psychology, 1958, $55,289.296$

BERLYNE, D. E. Conflict, arousal, and curiosity. New York: McGraw-Hill, 1960.

LECKART, B. T. Looking time: The effects of stimulus complexity and familiarity. Perception \& Psychophysics, 1966, 1, 142-144.

LECKART, B. T. Task specific decrements in the duration of attention, Psychonomic Science, 1967, 9, 559-560.

LECKART, B. T., \& BAKAN, P. Complexity judgments of photographs and looking time. Perceptual and Motor Skills, 1965, 21, 16.18.

WELKER, W. 1. Some determinants of play and exploration in chimpanzees. Journal of Comparative and Physiological Psychology, 1956a, 49, 84-89.

WELKER, W. I. Variability of play and exploratory behavior in chimpanzees. Journal of Comparative and Physiological Psychology, 1956b, 49, 181-185. WELKER, W. I. Effects of age and experience on play and exploration of young chimpanzees. Journai of Comparative and Physiological Psychology, $1956 \mathrm{c}, 49,223-226$.

\section{NOTE}

1. Address: Department of Psychology, Western Washington State College, Bellingham, Wash. 98225.

(Accepted for publication July 19, 1968.) 